Kirkilevsky S. I., Polyasny V. O., Mashukov A. O., Kovalevskaya L. A. Nonel approaches in the field of gastric cancer surgical

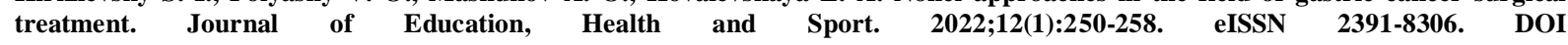
http://dx.doi.org/10.12775/JEHS.2022.12.01.021

https://apcz.umk.pl/JEHS/article/view/JEHS.2022.12.01.021

https://zenodo.org/record/5894561

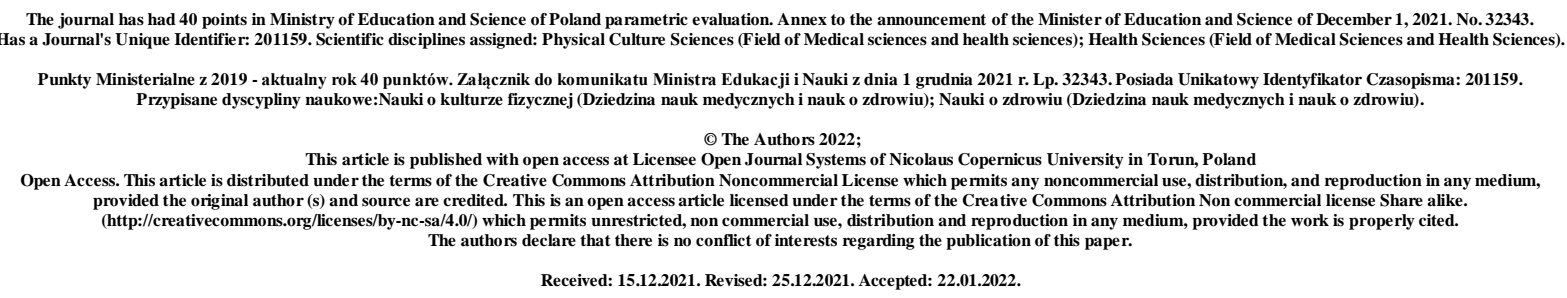

\title{
Nonel approaches in the field of gastric cancer surgical treatment
}

\author{
S. I. Kirkilevsky ${ }^{1}$, V. O. Polyasny ${ }^{2,3}$, A. O. Mashukov ${ }^{2,3}$, L. A. Kovalevskaya ${ }^{2}$ \\ ${ }^{1}$ Oncological clinic "Target", Kyiv, Ukraine \\ ${ }^{2}$ International Humanitarian University, Odesa, Ukraine \\ ${ }^{3}$ CNI "Odessa Regional Oncology Center", Odesa, Ukraine
}

Mashukov Artem Alekseevich, Odessa, Dobrovolsky Avenue, 75, apt. 4, mashukster@gmail.com

Abstract

The aim of the study: to estimate the impact of different cellular phenotype of gastric tumors on the survival of patients treated according to standard protocols.

Object and methods of research.The work presents the results of a study conducted on the basis of two medical institutions - the National Cancer Institute and the abdominal oncology and surgery department of the Odessa Regional Oncology Center. 221 patients underwent surgery for gastric cancer (GC) in the period 2007-2013. The average age of patients was $60.88 \pm 10.5$ years, men -180 , women -41 . Of the total number of operated, 143 patients underwent surgery in the amount of gastrectomy, 78 - subtotal distal resection.

Results. All patients included in the study underwent radical surgery. Tumors removed during surgery were examined using histological, cytological, immunohistochemical methods and genetic tests. The obtained results are monitored for the presence/absence of clinical parallels and mathematical correlations. It was concluded that some combinations of immunohistochemical (IHC) markers were effective in separating groups of patients with 
different biology of gastric cancer.

Conclusions. Performing more advanced operations (including the use of D2 lymph dissections) is the most effective option for surgical treatment of microsatellite-unstable and Epstein-Barr virus-associated gastric cancer.

Keywords: gastric cancer; oncoproteins; immunohistochemical study; new classification.

\section{Introduction}

The problem of combating cancer is one of the most pressing and unresolved nowadays. Every year, more than 12 million people in the world are diagnosed with cancer for the first time, and about 7 million die from this disease. This fully applies to gastric cancer (GC). Treatment of this pathology remains an urgent problem due to its high prevalence and aggressiveness. Stably high mortality is characteristic of gastric cancer, its level ranks third among the world in both men and women, second only to mortality from lung cancer.

In Ukraine, according to the latest statistics, in the majority of first-time patients (7669 cases), stage III-IV disease $(68.8 \%)$ is established at the initial treatment. At the same time, only $45.5 \%$ of patients were covered by special treatment, and $58.1 \%$ did not survive one year from the moment of diagnosis.

From the above data it is possible to see that gastric cancer refers to diseases that are very often detected at a late stage, when surgical treatment is usually impractical or can not be performed for technical reasons. In the case when the high prevalence of the tumor process still allows you to perform surgery in the amount of resection and gastrectomy, the prognosis is quite problematic.

It follows that despite the performance of surgical interventions in significant volumes (combined and extended operations, D2, D3 lymph dissection), the use of modern drug therapy regimens, indicators characterizing the effectiveness of such treatment (average life expectancy, cancer-specific survival) leave wish for the best.

It should be noted that according to world experience, tactics to combat cancer in general and gastric cancer in particular by improving only the methods of combined and comprehensive treatment is ineffective. The main attention today should be attracted to the introduction of modern diagnostic methods.

Common forms of medical management in gastric cancer patients involve the use of combined and multi-organ surgery $[14,17,21]$. In this case, in addition to local decease control achievement, as a rule, there might be a massive lymphogenic metastasis to the 
regional lymph nodes (LN). In this regard, affected organ directed surgery always includes dissection of various groups of lymph nodes, defined as lymph dissection in the amount of D2 or D3. Standard D2 dissection in radical surgery reduces mortality and increases five-year survival. Lymph dissection in the volume of D3 with removal of paraaortic nodes tends to be controversial among oncosurgeons in the view of its possible complications [22]. It is well known so-called "gap" or "dissociation" between so called eastern and western approaches to extend operations in the light of clinical data, and up to this time enlarged lymphatic dissections seems to be conditional in some regions of the world. While lymphatic dissections for gastric cancer treatment have become the standard of surgical treatment in Japan since the early 1990s, controversial attitude to them has continued in Europe and North America up to the late 2000s. For example, to the German national surgical guidelines, D2 lymph dissection has been entered only in 2010. As involuntary observers, and in some ways participants in present global discussion, in the paper authors ask a simple question: might it be only different variations of tumor biology because of prevailing various genetic stomach cancer subsets in different countries?

Example of such a well-known British study MAGIC, which has become a classic and even encyclopedic, nethertheless is still has been criticized for the fact that $40 \%$ of patients was not underwent D2 lymph dissection at all. The question arises whether it is possible to explain such a well-known fact by the different ratio of genetic and biological parameters in the studied populations in the groups of so-called "molecularly different" gastric cancer subset $[12,13] ?$

In the presented work the results of own researches of surgical and pathohistological parallels are resulted. All patients included in the study were operated on, the tumor, removed during surgery, was examined using histological, cytological, immunohistochemical methods and genetic tests. The results obtained in terms of the presence and absence of statistical parallels and mathematical correlations between the studied markers are also monitored.

The use of complex therapy in order to improve long-term results, includes, in addition to surgical treatment, the use of several anticancer drugs. In general, the effect of such treatment, according to literature sources, increases survival to 40-50\%. At the same time, there are publications in which, unfortunately, in patients with stage III of gastric cancer, the effect of complex therapy was not achieved $[15,16]$.

Many factors affect the quantitative and qualitative indicators of life of treated patients. To date, there is no instrument or device that could measure the life expectancy of a particular person. However, based on the mathematical analysis of many parameters at once, it is 
possible to predict the life expectancy of the patient, including gastric cancer. It depends on a large number of factors that may be changed during the development of the disease in a particular patient.

Our task was to track such trends and knowing the patient's age, stage of the disease, indicators of the degree of aggressiveness of the tumor be capable to make an approximate forecast of future life expectancy after surgery. This did not take into account possible other causes of death of the patient, which are not related to cancer (heart attack, stroke, etc.). The need for such mathematical tools in routine clinical work is long overdue. Therefore, the search for new ways to assess the prognosis of gastric cancer in each individual patient with gastric cancer is extremely modern and relevant.

The aim of the study: to study the influence of the molecular phenotype of gastric tumors on the survival of patients who underwent radical surgery.

Object and methods of research. The study has included 221 patients operated on for gastric cancer. The average age was $60.88 \pm 10.5$ years, men -180 , women -41 . A total of 143 gastrectomies and 78 distal subtotal resections were performed. The study was designed to form four groups of patients with gastric cancer, who underwent various combinations of advanced and combined surgery: a combination of D2 and multivisceral resection (MVR) - 26 patients, only D2 without MVR - 36 patients, a combination of D1 and MVR - 74 patients, only D1 without MIA - 85 patients. The study was one-centered, retrospective.

Three criteria were used to assess the effectiveness of the treatment method: average life expectancy in the group; survival curves indicating differences with the base group $(p<0.05)$. Also, in some cases, we compared the areas under the survival curves. This approach was more effective than estimating only life expectancy. It also made it possible to estimate the number of patients who survived this period. Statistical processing of the obtained data was performed using nonparametric methods (Spearman's rank correlation coefficient - rS), Wilcoxon's criterion (homogeneity of the studied features). 5-year survival was studied by the method of constructing a model of proportional risks for Cox.

As noted, the comparison of survival curves of radically operated patients with cervical cancer with different combinations of IHC markers created many new groups of patients with different life expectancy. However, when using a comparison of 1 marker, such as $\mathrm{p} 53$, the survival curves merged so that it was not even necessary to calculate the Student's t-test (Fisher, Wilcoxon, etc.) to understand that this is one study group.

Finding groups that differ from each other is one of the goals of any scientific search. The design axiom of our study was the following statement: "One group has the same life 
expectancy."

That is, the opposite logical statement is true: if two (three, four, five, etc.) groups did not differ, then they are not two groups, but one. The statement is completely synonymous with the concept of testing the hypothesis of calculations: $\mathrm{H}_{\mathrm{o}}$ - if a and $\mathrm{b}$ do not differ $(\mathrm{p}>$ 0.05) and $\mathrm{H}_{1}-\mathrm{a}$ and $\mathrm{b}$ differ from each other $(\mathrm{p}<0.05)$. If we denote the existence of group $\mathrm{a}$, independent of group $b$, and survival by the letter $S$ (survival), then $a=S_{1}, b=S_{2}$. That is, it will be two different survival curves. The general concept in this case could be as follows: "any new oncological classification can be such if it creates groups of patients with independent, different from others survival."

Focusing on the combination of markers [11], we identified four groups of patients who are promising in terms of impact on survival in certain surgical procedures: 1) p53-, VEGFR+, her2/new+, Ki-67+, diffuse cancer; 2) p53+, VEGFR-, her2/new +, intestinal form of gastric cancer; 3) p53+, VEGFR-, her2/ new -, mucus-producing form, "noncardiac cancer \#1"; 4) p53-, VEGFR-, her2/ new +, "noncardiac cancer \#2" (number of groups is presented in table 2).

The groups were largely similar and corresponded to the TCGA classification [23], but also had much in common with the ACRG classification [24], which emphasizes the determination of p53 and manifestations of microsatellite instability. Additionally, patients were examined for markers CDH1, as well as MLH1, MLH3, MSH2, MSH6. The BRCA-1 marker at GC was considered as a candidate for the creation of the 5th group. At the same time, no mutations in the BRCA-1 5382insC gene were found in 10 patients who underwent real-time PCR sequencing of this marker, which did not allow to isolate these patients in a separate study group.

Table 1

Distribution of genetic groups depending on the type of surgery

\begin{tabular}{|c|c|c|c|c|c|}
\hline \multirow{2}{*}{$\begin{array}{c}\text { Type of operation } \\
\text { performed }\end{array}$} & \multicolumn{4}{|c|}{ Group, $\mathrm{n}(\%)$} & \multirow{2}{*}{ Total } \\
\cline { 2 - 5 } & 1st & 2nd & 3rd & 4th & 36 (16.29) \\
\hline $\begin{array}{c}\text { GE+D2, without } \\
\text { MVR }\end{array}$ & $13(5.88)$ & $5(2.26)$ & $2(0.91)$ & $16(7.24)$ & $26(11.77)$ \\
\hline $\begin{array}{c}\text { GE+D2, in } \\
\text { combination with } \\
\text { MVR }\end{array}$ & $10(4.53)$ & $9(4.07)$ & $2(0.91)$ & $5(2.26)$ & \\
\hline $\begin{array}{c}\text { GE without D2 and } \\
\text { MVR }\end{array}$ & $24(10.86)$ & $16(7.24)$ & $23(10.41)$ & $22(9.96)$ & $85(38.46)$ \\
\hline $\begin{array}{c}\text { GE+ MVR without } \\
\text { D2 }\end{array}$ & $8(3.62)$ & $39(17.65)$ & $18(8.15)$ & $9(4.07)$ & $74(33.48)$ \\
\hline Total & $55(24.89)$ & $69(31.22)$ & $45(20.36)$ & $52(23.53)$ & $221(100.0)$ \\
\hline
\end{tabular}




\section{Research results}

1. 4 biological types of gastric cancer have been experimentally derived, which respond differently to the application of the program of individualization of surgical treatment. Type 1 has the following combination of markers: p53-, VEGFR+, her2/new,$+ \mathrm{Ki}-$ 67+, diffuse cancer; Type 2 combination of markers: p53+, VEGFR-, her2/new +, intestinal form of gastric cancer; Type 3 combination of markers: p53+, VEGFR-, her2/new -, mucus production, "I ${ }^{\text {st }}$ noncardiac cancer"; Type 4 combination: p53-, VEGFR-, her2/new +, and "II nd noncardiac cancer".

2. It was found that the implementation of extended and combined operations increased the survival of patients with diffuse gastric cancer, which we classify as type \#1. The median survival of this group was 7 months. The differences between the survival curves were also statistically significant; $\mathrm{p}<0.05$.

3. With gastric cancer of the 2nd type, the survival of patients with MVR was improved the most (48 out of 69 patients). This number was enough to sum up. The median survival of this group was 20.5 months. The obtained data indicate that the implementation of MVR increases the survival of patients with type 2 gastric cancer with a probability of $95 \%$; $\mathrm{p}<0.05$.

4. The highest overall survival was characteristic of patients with type 3 gastric cancer in the case of combined and multi-organ resections. This category of patients in our study was close to microsatellite-unstable cancer, which is promising for surgical treatment in the locally advanced stage.

The aim of the analysis of patients with verified gastric cancer who were operated on in 2007-2014 was to show that the standard criteria for assessing and predicting the future life expectancy of these patients do not quite fit into existing methods of assessing biological behavior. For example, it is believed that the greater efficiency of extended lymphatic dissections in patients with gastric cancer is due to the prevalence of so-called intestinal form of stomach cancer according to Lauren (1965), for which lymphogenic metastasis is more likely than hematogenous. Diffuse forms of gastric cancer predominate in Europe and North America. That is why the Japanese experience of extended dissections has not been fully confirmed by all researchers.

Another variant of explanation taking into attention underlines why the extended lymph dissection was more effective in Japan and South Korea than in Europe and North America. For example, the higher percentage of Nakayama surgery (direct esophago- 
duodenoanastomosis after gastrectomy) is due to the morphological features of the body structure of the Asian race. In Europeans, due to greater dolichomorphism, this is possible relatively rarely. Our own Nakayama operation experience includes 9 operations. In the same way, the greater efficiency of a certain type of operation may be accompanied by other tumor genetics - the presence of a different percentage of molecular GC subtypes. In this case, the principle of occult individualization may work.

Based on the use of the developed genetic and morphological classification of gastric cancer based on the introduction of a panel of markers, their predictive significance was revealed, and tactical conclusions on radical gastric cancer surgery were proposed. The highest survival rate of patients who underwent combined surgery and multivisceral resection is significantly inherent in patients with intestinal gastric cancer. Advanced surgeries, including those performed with D2 lymph dissection, significantly increase the overall survival of patients in the groups of diffuse cancer and non-cardiac cancer II $^{\text {nd }}$. The positive predictive significance of radical resections in patients with locally advanced gastric cancer with form $\mathrm{I}^{\text {st }}$ non-cardiac cancer has been established.

\section{Conclusions}

1. The influence of "biology" of gastric cancer on the results of surgical treatment was studied with the help of groups of molecular markers.

2. In order to detail the biological aggressiveness of the tumor, determine the prognosis of the disease and develop personalized tactics of adjuvant CT and target treatment, it is advisable in the postoperative period to determine the genetic variant of gastric cancer.

3. Groups of patients with gastric cancer with different IHC phenotype have different survival, which creates a basis for the formation of a new molecular classification of gastric cancer.

4. There are 4 biological types of gastric cancer, which respond differently to the application of the program of individualization of surgical treatment. Type 1 is characterized by the following combination of markers: p53-, VEGFR+, her2/ new +, Ki-67+, diffuse cancer; at type 2 the combination of markers is defined: p53+, VEGFR-, her2/new + , the intestinal form of GC; Type 3 has a combination of markers: p53 +, VEGFR-, her2/new-, often mucus production, "I ${ }^{\text {st }}$ noncardiac cancer"; at type 4 the combination of markers is found: p53-, VEGFR-, her2/new +, «II ${ }^{\text {nd }}$ noncardiac cancer». 


\section{References}

1. Coppedè F, Lopomo A, Spisni R, et al. Genetic and epigenetic biomarkers for diagnosis, prognosis and treatment of colorectal cancer. World $\mathrm{J}$ Gastroenterol 2014;20(4):943-56. doi: 10.3748/wjg.v20.i4.943.

2. He D, Zhang YW, Zhang NN, et al. Aberrant gene promoter methylation of p16, FHIT, CRBP1, WWOX, and DLC-1 in Epstein-Barr virus-associated gastric carcinomas. Med Oncol. 2015;32(4):92. doi: 10.1007/s12032-015-0525-y.

3. Malcolm RA. The Cancer Handbook. 2nd ed. Wiley-Blackwell; 2007. 1616 p.

4. Scott FG. Commentary: 'The Epigenotype' by CH Waddington. Int J of Epidemiol. 2012;41(1):20-23. doi: 10.1093/ije/dyr186.

5. Sheffield BS, Garratt J, Kalloger SE, et al. HER2/neu testing in gastric cancer by immunohistochemistry: assessment of interlaboratory variation. Arch Pathol Lab Med. 2014;138(11):1495-1502. doi: 10.5858/arpa.2013-0604-OA.

6. Vollan HKM., Caldas C. The Breast Cancer Genome -A Key for Better Oncology. BMC Cancer. 2011;11:501. doi: 10.1186/1471-2407-11-501.doi: 10.1186/1471-2407-11-501.

7. Baryshev AG. Long-term results of treatment of patients with gastric cancer in the light of the biomolecular features of the tumor. Ros. biotherapeutic magazine 2011;2:85-8.

8. Vasilenko IV, Gulkov YuK, Zaporozhchenko NV. Histological heterogeneity of gastric cancer. Ukr. morphologist. almanac. 2011;9(1):26-31.

9. Danilova IV. On the issue of clinical and morphological variants of gastric cancer. Archive pathologist. 2005;67(5):324.

10. Imyanitov EN. Controversial aspects of HER2 diagnostics. Modern oncol. 2010;31:5-19.

11. Weiguo C, Rong F, Weiping Y, et al. VEGF-C expression is associated with the poor survival in gastric cancer tissue. Tumor Biology. 2014;35(4):3377-83.

12. Yamazaki K, Tajima Y, Makino R, et al. Tumor differentiation phenotype in gastric differentiated type tumors and its relation to tumor invasion and genetic alterations. World J of Gastroenterol. 2006;12(24):3803-9. doi: 10.3748/wjg.v12.i24.3803

13. Hwang J E, Hong J Y, Kim J E, et al. Prognostic significance of the concomitant existence of lymphovascular and perineural invasion in locally advanced gastric cancer patients who underwent curative gastrectomy and adjuvant chemotherapy. Jpn J Clin Oncol. 2015 Jun;45(6):541-6. doi: 10.1093/jjco/hyv031 
14. V'yun SV. Surgical tactics of treatment of ailments for cancer of the duct in the fallow stage of the risk of obstruction of the stravochido-intestinal anastomosis. Ukr. magazine medicine, biology and sports. 2018;3(7):84-9. doi:10.26693/jmbs03.07.084

15. Chernousov A, Khorobrykh T, Vychuzhanin D, et al. Intra-abdominal chemotherapy in the combined treatment of advanced gastric cancer. Doctor. 2015;2:28-31.

16. Kotov AA. Immediate results of adjuvant chemoradiotherapy for locally advanced gastric cancer. Bulletin of VSMU. 2017;3:63-72.

17. Lazirsky VO. Surgical tactics of exalting ailments to reduce the incidence of breast cancer. Ukr. magazine medicine, biology and sports. 2017;5:82-6.

18. Skoropad VYu, Berdov BA, Galkin VN. Neoadjuvant chemoradiotherapy followed by D2 gastrectomy for gastric cancer: treatment outcomes, molecular biological predictors of prognosis. Eurasian oncologist. magazine 2016;4(2):408.

19. Levik EN, Kolesnik AP, Kuzmichev AA, et al. Evaluation of long-term results of surgical and complex treatment of patients with gastric cancer. Actual. pharmaceutical nutrition i honey. science and practice. 2012;2:116-8.

20. Borovskaya TF, Zenyukov AS, Nikulin MP, [et al.] Prognostic value of lymphogenous micrometastases in gastric cancer. Ros. oncologist. magazine 2011;3:24-8.

21. Tarasov VA, Vinogradova MV, Klechikov VZ, et al. Surgical treatment of common forms of gastric cancer. Practical oncology. 2001;7:52-8.

22. Shanazarov NA, Arybzhanov DT, Saburov AR. Surgical treatment of gastric cancer: a review of the literature. Honey. Bulletin of Bashkortostan. 2010;5(3):123-8.23.

Zhou L, Huang W, Yu HF, et al. Exploring TCGA database for identification of potential prognostic genes in stomach adenocarcinoma. Cancer Cell Int. 2020 Jun 23;20:264. doi: 10.1186/s12935-020-01351-3.

24. Wang Q, Liu G, Hu C. Molecular Classification of Gastric Adenocarcinoma. Gastroenterology Res. 2019 Dec;12(6):275-82. doi: 10.14740/gr1187. 